\title{
La reforma local en la Comunidad Autónoma del País Vasco: la necesidad perentoria de una Ley municipal de Euskadi (1)
}

Martín María Razquin Lizarraga

\begin{abstract}
Sumario: I. Las competencias de la Comunidad Autónoma del País Vasco sobre Administración Local.- - II. El punto de partida: la regulación antes vigente._III. La reforma local de 2013.-IV. La singularidad de los territorios históricos. - - V. La aplicación de la reforma local en la Comunidad Autónoma del País Vasco. V.1. Las disposiciones de la Irsal relativas a la Comunidad Autónoma del País Vasco. V.2. Nuevas cláusulas generales de salvedad: la disposición adicional $1 .^{a}$ de la LRSAL y la disposición adicional 8. ${ }^{a}$ del TRLHL. V.3. Actualización de denominaciones de los territorios históricos. V.4. Las competencias de los municipios. V.5. La estructura local: otras entidades locales, regímenes especiales y concejos. V.6. Tutela financiera. V.7. Funcionarios con habilitación de carácter nacional. V.8. Retribuciones de cargos, personal eventual y funcionarios. - VI. Papel de las instituciones comunes y de los territorios históricos en el desarrollo de la reforma local._VII. ¿Por fin, una Ley municipal de Euskadi?
\end{abstract}

\section{Las competencias de la Comunidad Autónoma del País Vasco sobre Administración Local}

Las competencias de la Comunidad Autónoma del País Vasco sobre Administración Local se encuentran recogidas en su Estatuto de Autonomía de 1979 (en adelante EAPV). El EAPV diseña un singular modelo de articulación competencial interna, puesto que nos encontramos, por un lado, con las Instituciones comunes de la Comunidad Autónoma (Parlamento Vasco y Gobierno Vasco) y, por otro, con los Territorios Históricos (Juntas Generales y Diputaciones Forales). Es un sistema singular, que se enraiza en la disposición adicional 1. ${ }^{a} \mathrm{CE}$, garantía de la foralidad.

El artículo 10 EAPV establece una competencia exclusiva de la Comunidad Autónoma del País Vasco de carácter general sobre el régimen local en los siguientes términos:

(1) Este homenaje a mi queridísimo amigo, colega y paisano, Demetrio Loperena Rota, no podía rehuir el análisis de la materia de la Administración Local. Nuestra última colaboración escrita se manifestó en el libro colectivo sobre Derecho Local de Navarra, editado por lustel en el año 2013. Es, por ello, que ahora en recuerdo a su asentamiento en Donostia-San Sebastián he tomado como objeto de estudio el régimen local vasco. 
«Régimen Local y Estatuto de los Funcionarios del País Vasco y de su Administración Local, sin perjuicio de lo establecido en el artículo 149.1.18. ${ }^{a}$ de la Constitución» (apartado 4).

Como puede verse, se trata de una competencia que se define como exclusiva y que alcanza a toda la materia "régimen local», aunque debe respetar el marco general establecido en el artículo 149.1.18. ${ }^{a}$, que alberga la materia de régimen jurídico de la Administración Local. Es, por ello, que le es aplicable la legislación básica del Estado, pero sin perjuicio de las competencias derivadas de sus derechos históricos, en virtud de la garantía institucional del régimen foral vasco reconocida en la disposición adicional $1 .{ }^{a}$ CE.

Por otra parte, el artículo 10 EAPV contiene otro apartado 1, referido a un aspecto parcial del régimen local, las «demarcaciones territoriales municipales».

Asimismo, el EAPV al referirse a las instituciones de los Territorios Históricos especifica sus competencias en una triple clasificación:

a) Expresas, recogidas en el Estatuto de Autonomía: entre las que se encuentran las establecidas en el artículo 37, letras c) «Demarcaciones territoriales de ámbito supramunicipal que no excedan los límites provinciales», d) "Régimen de los bienes provinciales y municipales, tanto de dominio público como patrimoniales o de propios y comunales», y e) «Régimen electoral municipal».

b) Competencias que les sean transferidas.

c) Competencias de desarrollo normativo y ejecución en las materias que señale el Parlamento Vasco.

Finalmente, debe tenerse en cuenta el artículo 41 EAPV que consagra el sistema foral tradicional de Concierto Económico para las relaciones de orden tributario y financiero entre el Estado y el País Vasco, plasmadas en la Ley 12/2012, de 23 de mayo.

\section{El punto de partida: la regulación antes vigente(2)}

La regulación vigente con anterioridad a la reforma introducida en 2013 se fundaba en dos cuerpos normativos diferenciados. Por un lado, la Ley reguladora de las Bases de Régimen Local de 1985 (en adelante LRBRL) que contenía la regulación básica estatal y además se refería en dos preceptos de forma específica a la Comunidad Autónoma Vasca. El artículo 39 (3) salvaba el régimen "peculiar» de los Territorios Históricos, lo que venía ratificado por lo dispuesto en la disposición adicional 2. ${ }^{a}$ (4).

Pero, además, esta disposición adicional 2. ${ }^{a}$ se refería a algunos aspectos de índole municipal. En concreto, la salvedad de la aplicación de la LRBRL se centraba en dos materias:

(2) Véase J. M. ENDEMAÑO ARÓSTEGUI, "El municipio en la Comunidad Autónoma Vasca», en lura Vasconiae, núm. 2/2005, 125-148, quien examina la regulación estatal y ofrece datos sobre la situación municipal en Euskadi.

(3) Véase el comentario a este artículo 39 efectuado por E. COBREROS MENDAZONA, en el libro colectivo dirigido por M. REBOLLO PUIG y M. IZQUIERDO CARRASCO, Comentarios a la Ley Reguladora de las Bases de Régimen Local, Ed. Tirant lo blanch, Valencia, 2006, pp. 907-920.

(4) Véase el comentario a esta disposición adicional de E. COBREROS MENDAZONA, en el libro colectivo ya citado, Comentarios a la Ley Reguladora de las Bases de Régimen Local, pp. 3778-3790. 
a) El régimen económico-financiero de los municipios: que se aplicará en los términos previstos en el Concierto Económico y sin que el nivel de autonomía de los municipios de los Territorios Históricos sea inferior al de los municipios del resto del Estado (apartado 6).

b) La regulación de los funcionarios de habilitación de carácter nacional: determinadas competencias se sustraen al régimen general, tales como convocatoria de concursos, nombramiento de funcionarios, fijación de méritos del baremo en un 10 por ciento por la Comunidad Autónoma y en un 25 por ciento por la corporación local interesada, y formación de estos funcionarios (apartados 7 (5), 8 y 9) (6).

La singularidad de la disposición adicional 2. ${ }^{a}$ LRBRL fue declarada por el Tribunal Constitucional en su STC 214/1989, de 21 de diciembre, con motivo del examen de los preceptos relativos a los funcionarios con habilitación de carácter nacional ( $F$. J. 26). El Tribunal Constitucional afirmó que «la L. R. B. R. L., en la Disposición adicional 2. ${ }^{\text {a }}$, no ha hecho, pues, sino atender al propio mandato constitucional, preservando la singularidad misma de ese régimen foral en los aspectos organizativos, en unos términos y con un alcance perfectamente compatible con la propia Constitución». Añadió también que la garantía institucional del régimen foral comporta un tratamiento normativo singular propio de ese régimen local y ello aun frente a los poderes centrales del Estado, como ocurre en las competencias sobre los funcionarios con habilitación de carácter nacional.

La reforma efectuada por la Ley 7/2007, de 12 de abril (EBEP), recoge la regulación de los funcionarios con habilitación estatal en su disposición adicional 2. ${ }^{a}$. A dicha Ley se incorporó una disposición adicional 3 . $^{a}$ cuyo segundo párrafo contenía la salvedad de la Comunidad Autónoma del País Vasco, y además de forma más precisa la relativa a los funcionarios con habilitación de carácter estatal (7).

El segundo bloque normativo se encuentra en la Ley de Haciendas Locales 39/1988, de 28 de diciembre y su posterior Texto Refundido de la Ley de Haciendas Locales de 2004 (en adelante TRLHL). Su artículo 1.2 dispone que la Ley de Haciendas Locales «se aplicará en todo el territorio nacional, sin perjuicio de los regímenes financieros forales de los Territorios Históricos del País Vasco y Navarra». Este artículo 1.2 ha mantenido su redacción desde la aprobación inicial de la Ley en 1988, luego se recogió en el texto refundido y sigue inalterado en la actualidad, no habiendo sido reformado por la LRSAL.

(5) Este apartado 7 es el único que ha sufrido modificación desde 1985. Fue modificado por la Ley 57/2003, de 16 de diciembre, con el único efecto de cambiar la referencia del "artículo 99.2 párrafo primero" por la del «artículo 99.1».

(6) Debe tenerse en cuenta que los artículos 92, 98 y 99 LRBRL fueron derogados por la Ley 7/2007, de 12 de abril, del Estatuto Básico del Empleado Público, que dio nueva regulación a los en ella denominados funcionarios con habilitación de carácter estatal (disposición adicional 2. ${ }^{a}$ ). Ahora la LRSAL ha derogado tanto esta disposición adicional $2 .^{a}$ como la disposición transitoria 7. ${ }^{\mathrm{a}}$ EBEP.

(7) En concreto el párrafo 2. ${ }^{\circ}$ del apartado 2 de esta disposición adicional 3. ${ }^{a}$ del EBEP dice así: "En el ámbito de la Comunidad Autónoma del País Vasco el presente Estatuto se aplicará de conformidad con la disposición adicional primera de la Constitución, con el artículo 149.1.18. ${ }^{a}$ de la Constitución y con la Ley Orgánica 3/1979, de 18 de diciembre, por la que se aprueba el Estatuto de Autonomía para el País Vasco. Las facultades previstas en la disposición adicional segunda respecto a los funcionarios con habilitación de carácter estatal serán ostentadas por las Instituciones Forales de sus territorios históricos o por las Instituciones Comunes de la Comunidad Autónoma, en los términos que establezca la normativa autonómica». 
Pero, además, la LHL de 1988 incorporó una disposición adicional 18. ${ }^{a}$ referida a los Territorios Históricos del País Vasco en relación con el régimen económico financiero en materia municipal, que constituye la copia en esta sede legal del apartado 6 de la disposición adicional $2 .^{a}$ de la LRBRL. EI TRLHL la renumeró como disposición adicional 8. ${ }^{\mathrm{a}}$.

Por su parte, la Ley Orgánica 2/2012, de 27 de abril, de Estabilidad Presupuestaria y Sostenibilidad Financiera (en adelante LOEPSF), recoge en su disposición final 3. ${ }^{a}$, relativa a las Haciendas Forales, la salvedad del régimen especial de la Comunidad Autónoma del País Vasco. Así el apartado 2 de esta disposición dice: «En virtud de su régimen foral, la aplicación a la Comunidad Autónoma del País Vasco de lo dispuesto en esta Ley, se entenderá sin perjuicio de lo dispuesto en la Ley del Concierto Económico» (8).

En desarrollo de esta LOEPSF se ha dictado la Orden HAP/2105/2012, de 1 de octubre, sobre obligaciones de información, en cuya disposición adicional única se señala que su aplicación en la Comunidad Autónoma del País Vasco lo será de conformidad con la disposición adicional 3. ${ }^{a}$ de la LOEPSF y sin perjuicio de las especialidades derivadas del régimen foral.

En desarrollo de esta previsión, el Territorio Histórico de Gipuzkoa ha aprobado la Norma Foral 1/2013, de 8 de febrero, por la que se desarrolla la competencia de tutela financiera en materia de estabilidad presupuestaria y sostenibilidad financiera de las entidades locales de Gipuzkoa (publicada en el BOG de 12 de febrero de 2013).

Así pues, el punto de partida era muy claro: a) singularidad absoluta del régimen jurídico de los Territorios Históricos; b) algunas salvedades (muy pocas) sobre el régimen municipal; y c) una gran salvedad en materia económico-financiera y presupuestaria, en especial, en la tutela financiera sobre los municipios.

\section{La reforma local de 2013}

El Boletín Oficial del Estado del día 30 de diciembre de 2013 publica la Ley 27/2013, de 27 de diciembre, de racionalización y sostenibilidad de la Administración Local (en adelante LRSAL).

Se trata de una Ley que introduce una profunda reforma en el régimen local español, seguramente la más relevante desde la aprobación de la LRBRL. La LRSAL modifica un importante número de preceptos de la $L R B R L$, así como algunos del TRLHL. Incide en aspectos sustanciales de la Administración Local, aunque no supone una reforma directa del mapa local, sí lo hace de forma indirecta al modificar el sistema de competencias municipales y provinciales.

La LRSAL se funda en la reforma del artículo 135 CE y su desarrollo por la LOEPSF, disposiciones todas ellas que se insertan asimismo dentro de las relevan-

(8) B. PÉREZ DE LAS HERAS y S. LARRAZÁBAL BASAÑEZ recomiendan la modificación del Capítulo II de la Ley 12/2002 del Concierto Económico para desarrollar su parte financiera y adecuarla a la nueva regulación de la LOEPSF («Control presupuestario en la Unión Europea, reforma constitucional, ajuste de los presupuestos de las Administraciones Públicas y su impacto en el autogobierno vasco en el marco del Concierto Económico», en Revista Vasca de Administración Pública núm. 94/2012, p. 174). 
tes reformas acaecidas en el Derecho de la Unión Europea y principalmente en la aprobación del Tratado de Estabilidad, Coordinación y Gobernanza de la Unión Económica y Monetaria de 2 de marzo de 2012, que ha entrado en vigor el día 1 de enero de 2013 (9).

La reforma local que se efectúa es de gran calado y profundidad(10) por más que una ligera lectura pudiera hacer pensar, erróneamente, que se trata de una nueva modificación de las leyes sobre Administración Local, de corte similar a las realizadas con anterioridad. De forma indirecta, constituye una reconstrucción del Estado, principalmente del nivel local (municipios y provincias), pero también con afecciones a las Comunidades Autónomas.

Los principios que mueven la reforma son los constitucionales, el de autonomía local, al que se añade ahora la exigencia de equilibrio presupuestario del artículo 135 CE.

La relevancia de la reforma ha provocado una nueva intervención desde los territorios forales, en defensa de sus derechos históricos sobre régimen local. Desde el primer momento se ha estado atento a las modificaciones que se pretendían introducir, defendiendo la inclusión, frente a ellas, de nuevas excepciones fundadas en los derechos históricos.

El Anteproyecto de LRSAL, en su versión de 24 de mayo de 2013 sometida al Consejo de Estado, incluía una disposición adicional $1 .{ }^{a}$ referida a los "Regímenes y Haciendas forales", que pretendía salvaguardar los derechos históricos. Esta disposición pasó luego al proyecto de ley publicado en el BOCG, Congreso de los Diputados, de 6 de septiembre de 2013. Su primer apartado se refería a la Comunidad Foral de Navarra y el segundo a la Comunidad Autónoma del País Vasco, y ambos tenían una redacción muy similar, prácticamente equivalente.

Sin embargo, en el texto definitivo de la LRSAL aparecen grandes cambios puesto que se separa la regulación en dos disposiciones adicionales (una para Navarra y otra para el País Vasco) con contenido bien diferente y, además, se modifican la disposición adicional 2. ${ }^{a}$ de la $L R B R L$ y la disposición adicional 8. ${ }^{a}$ del TRLHL en relación con la Comunidad Autónoma Vasca (11).

(9) F. J. VILLAR ROJAS ha advertido que las razones alegadas para la reforma local hacen referencia a la crisis económico-financiera, pero la regulación aprobada obedece más bien a otros objetivos, porque «ni la deuda ni el déficit público locales tienen suficiente peso como para imponer la reforma puesta en marcha» («Razones y contradicciones de la Ley de Racionalización y Sostenibilidad de la Administración Local», en El Cronista del Estado Social y Democrático de Derecho núm. 46/2014, p. 44).

(10) Tan es así que el Ministerio de Hacienda y Administraciones Públicas ha emitido una «Nota explicativa de la Reforma Local», de fecha 5 de marzo de 2014, ante las numerosas dudas surgidas con motivo de la aplicación de la LRSAL.

(11) Es significativa la manifestación efectuada por del Senador del Grupo Parlamentario Vasco Sr. Cazalis Eiguren recogida en el Diario de Sesiones del Senado, Pleno, de 16 de diciembre de 2013. Afirma que se acepta y vota favorablemente el proyecto de ley dado que se han incluido en el trámite parlamentario las necesarias disposiciones para garantizar un mínimo del cumplimiento de su objetivo de preservar el peculiar sistema foral de régimen local, afirmando que el proyecto de ley remitido a las Cortes Generales no tiene demasiado en común con el texto que se aprueba en el Senado en lo que se refiere a la preservación del régimen foral, aunque enumera algunas cuestiones que han quedado aún sin resolver (pp. 8890-8891). 


\section{La singularidad de los territorios históricos (12)}

El EAPV establece una organización territorial singular dentro de la Comunidad Autónoma. Dicha organización territorial se deriva del respeto de los derechos históricos de los territorios forales (13), que no son simples provincias dirigidas por Diputaciones locales, sino que constituyen un elemento esencial de la propia Comunidad Autónoma.

Ya el Título Preliminar del EAPV se refiere a los Territorios Históricos para reconocer su singularidad (artículo 3). El artículo 24.2 EAPV los configura como poderes del País Vasco, y como tales les corresponde mantener sus instituciones forales y autoorganizarse, a cuyo efecto se rigen por su respectivo régimen jurídico privativo (artículo 37.1 EAPV). Y se les atribuye competencia exclusiva en ciertas materias expresamente citadas, así como el desarrollo normativo y ejecución en otras a determinar por ley del Parlamento Vasco (artículo 37, apartados 3 y 4). Además son los actores principales en el sistema foral tradicional de Concierto Económico, como se desprende claramente del artículo 41 EAPV.

En desarrollo de lo dispuesto en el EAPV, el Parlamento Vasco aprobó la Ley 27/1983, de 25 de noviembre, conocida como "Ley de Territorios Históricos" (en adelante, LTH), donde se articula el sistema de distribución de competencias a nivel interno entre las Instituciones comunes de la Comunidad Autónoma y los Órganos Forales de los Territorios Históricos y que fue examinada en la STC 76/1988, de 26 de abril.

Por otra parte, las Normas Institucionales de cada Territorio Histórico disciplinan, con absoluta autonomía, su organización propia y el funcionamiento de los órganos forales.

En definitiva, la singularidad de los Territorios Históricos, que tiene su entronque en la disposición adicional 1. ${ }^{a}$ CE, aparece diseñada en el EAPV y luego desarrollada en la LTH y en las Normas institucionales de cada uno de ellos. Ninguna duda se presenta a nivel interno, sobre su posición singular y sustancial dentro del ámbito autonómico vasco, creándose como cierre del sistema la Comisión Arbitral, órgano de resolución de conflictos entre ambos niveles territoriales (artículo 39 EAPV) (14).

La LRBRL de 1985 reconocía la singularidad de los Territorios Históricos de forma patente. Su artículo 39 es muy ilustrativo al efecto al afirmar que éstos conservan su régimen peculiar, aunque sean de aplicación supletoria las disposiciones de la LRBRL. Cabe advertir que este precepto no ha sufrido modificación alguna en la reforma de 2013.

Como ya he adelantado en otro lugar (15), los Territorios Históricos han ido dejando cada vez más de ser provincias para convertirse en partes estructurantes de la

(12) Para un examen más amplio de esta cuestión me remito a J. M. CASTELLS ARTECHE, "Los regímenes especiales de la Comunidad Autónoma del País Vasco", en Tratado de Derecho Municipal, cit., en especial, pp. 4379-4417.

(13) Dentro de la abundante bibliografía sobre esta cuestión, es de obligada cita T. R. FERNÁNDEZ RODRíGUEZ, Los derechos históricos de los territorios forales, Ed. CEC-Civitas, Madrid, 1985.

(14) Vid. al respecto los estudios de J. LEGUINA VILLA, "Los Territorios Históricos vascos: poderes normativos y conflictos de competencias", en Revista Española de Derecho Constitucional núm. 3/1981, pp. 63-92; y "La Ley 13/1994, de 30 de junio, reguladora de la Comisión Arbitral y el artículo 39 del Estatuto», en Revista Vasca de Administración Pública, núm. 41/1995, pp. 251-260; y el libro de E. COBREROS MENDAZONA, Escritos sobre la Comisión Arbitral, Ed. IVAP, Oñati, 2009.

(15) Me remito a mis trabajos "La preeminencia del carácter de Territorio Foral en la naturaleza dual de los Territorios Históricos. A propósito de la STS de 19 de mayo de 1997», en Revista Vasca de Administración Pública núm. 49/1997, pp. 275-300; y "Organización Foral y Hecho Diferencial», en el volumen Estado Autonómico y Hecho Diferencial de Vasconia, ed. IVAP y Eusko Ikaskuntza, San Sebastián, 2000, pp. 247-270. 
Comunidad Autónoma. Es, por ello, que el régimen previsto en la $L R B R L$ no les es aplicable ni siquiera con carácter supletorio(16), como puede verse en la reciente Sentencia del Tribunal Supremo de 20 de mayo de 2013 (RJ 2013/4685) que no admite la aplicación supletoria del artículo 63.1.b) LRBRL a favor de unos junteros que pretendían impugnar una Norma Foral de Bizkaia en vía contencioso-administrativa, y además rectifica su anterior doctrina de la sentencia de 12 de febrero de 2009.

Esta singularidad aparece asimismo plasmada en la disposición adicional 2. ${ }^{2}$ $L R B R L$, que establece importantes salvedades para su aplicación a los Territorios Históricos, con base en lo dispuesto en la disposición adicional 1. ${ }^{a} \mathrm{CE}$, tal como afirmó el Tribunal Constitucional en su Sentencia 214/1989, de 21 de diciembre.

Estas singularidades pueden agruparse en tres aspectos fundamentales:

1. Estructura y organización autónoma y propia de los Territorios Históricos, sin vinculación alguna a las disposiciones establecidas para los entes locales provinciales.

2. Reconocimiento de dos tipos de competencias bien diferenciadas: unas competencias como Territorios Históricos y otras derivadas de la asunción en su organización de las competencias de las Diputaciones provinciales (apartados 2, 3 y 4).

3. Reconocimiento del sistema financiero y tributario foral de Concierto Económico, así como el presupuestario y contable, manteniendo la exclusión expresa de la aplicación del artículo 92 bis LRBRL (funcionarios con habilitación de carácter nacional).

De todo ello se deriva que los Territorios Históricos cuentan con una potestad "normativa» también singular(17). Esta singularidad ha sido elevada al mayor rango mediante la Ley Orgánica 1/2010, de 19 de febrero, que somete las Normas Forales fiscales al exclusivo control del Tribunal Constitucional (18).

(16) COBREROS MENDAZONA considera incorrecto que se hable de aplicación supletoria, toda vez que muchas de sus disposiciones son excluidas radicalmente de su posible aplicación a los órganos forales de los Territorios Históricos (Comentarios a la Ley Reguladora de las Bases de Régimen Local, cit., p. 919).

(17) Al efecto es clarificador el estudio de R. JIMÉNEZ ASENSIO, "El sistema de fuentes del Derecho de la Comunidad Autónoma del País Vasco como "ordenamiento asimétrico»», en Revista Vasca de Administración Pública núm. 47/1997, v. II, pp. 127-171. Véase asimismo el estudio de L.I. GORDILLO PÉREZ y R. MÚGICA ALCORTA, "Introducción al sistema normativo foral de los Territorios Históricos: consideraciones y propuestas", en la obra coordinada por L. I. GORDILLO PÉREZ, El poder normativo de Bizkaia en un contexto multinivel y transnacional: retos y desafíos, 2012, pp. 15-66.

(18) Esta Ley Orgánica no ha estado exenta de críticas en la doctrina. Véanse los estudios de S. MUÑOZ MACHADO, "Crítica de la exclusión de las normas fiscales de los Territorios Históricos vascos del control jurisdiccional contencioso-administrativo", en Revista General de Derecho Administrativo núm. 29/1981; J. J. SOLOZÁBAL ECHEVARRÍA, "El blindaje foral en su hora. Comentario a la Ley Orgánica 1/2010», en Revista española de Derecho Constitucional núm. 30/2010, pp. 11-28; J. C. DUQUE VILLANUEVA, "Los procesos constitucionales de control de las normas fiscales vascas", en la misma Revista y número, pp. 29-71; y L. M. DÍEZ PICAZO, "Notas sobre el blindaje de las normas forales fiscales vascas», en Indret: Revista para el Análisis del Derecho núm. 3/2010, pp. 1-14. Por el contrario, se pronuncian sobre su constitucionalidad, E. LUCAS MURILLO DE LA CUEVA, "La reserva al Tribunal Constitucional del control de las normas fiscales vascas y la creación del conflicto en defensa de la autonomía local frente a leyes del Estado», en la obra colectiva dirigida por E. ALVÁREZ CONDE, El privilegio jurisdiccional de las normas forales fiscales vascas, Ed. Instituto de Dereco Público (Universidad Rey Juan Carlos I), Madrid, 2010, pp. 93 y ss; y C. PALAO TABOADA, "La constitucionalidad del régimen de control jurisdiccional de las normas forales fiscales", en Estudios Finacieros. Revista de Contabilidad y Tributación, núm. 370/2014, pp. 51-96. 
Los apartados antes comentados de la disposición adicional 2. ${ }^{a}$ LRBRL han sido confirmados en la reforma de 2013, por lo que la "singularidad" de los Territorios Históricos queda mantenida. Más aun, como se verá más adelante, los Territorios Históricos van a ver salvadas algunas competencias, en virtud de las nuevas disposiciones introducidas por las modificaciones efectuadas por la LRSAL.

Así pues, no hay más que una "reafirmación» foral.

Aparecen, asimismo, acrecentadas las competencias de los Territorios Históricos pertenecientes al «bloque provincial», que, como veremos, van a estar absolutamente teñidas por las salvedades reconocidas e incorporadas así, al menos parcialmente, al «bloque foral». Y ello porque las diputaciones provinciales quedan reafirmadas en la nueva LRSAL, pero siempre con el control autonómico y/o estatal, lo que provoca que, en el caso del País Vasco, los Territorios Históricos van a hacer "suyas» estas competencias sin dicho control externo por lo que las incorporan a su bloque competencial.

Basta comparar el nuevo contenido del artículo 36 LRBRL con el de su precedente para observar la ampliación de competencias a favor de las diputaciones provinciales. A lo que debe añadirse lo dispuesto en el artículo 26.2 LRBRL. Como señala el apartado 2 de la disposición adicional 2. ${ }^{a}$ LRBRL, los Territorios Históricos ejercerán las competencias asignadas a las diputaciones provinciales. No obstante, como luego se verá, algunas de ellas van a quedar "foralizadas" (incorporadas al «bloque foral») en virtud de lo dispuesto en las salvedades contenidas en la LRSAL así como por su relación con el Concierto Económico.

\section{La aplicación de la reforma local en la Comunidad Autónoma del País Vasco}

\section{V.1. Las disposiciones de la LRSAL relativas a la Comunidad Autónoma del País Vasco}

La LRSAL recoge las modificaciones relativas a la Comunidad Autónoma del País Vasco en diversas disposiciones. Por un lado, modifica la disposición adicional 2. ${ }^{a}$ LRBRL. Por otro, modifica asimismo la disposición adicional 8. ${ }^{a} \mathrm{TRLHL}$. Y en tercer lugar, añade una nueva disposición adicional 1. ${ }^{a}$ a la propia LRSAL. No se explica muy bien esta abundancia de disposiciones y menos el entrecruzamiento que se observa entre ellas, pero en todo caso persiguen un mismo objetivo: establecer las singularidades de la Comunidad Autónoma Vasca tanto en el nivel de los Territorios Históricos como en el municipal, y tanto respecto de la regulación general del régimen local como en la relativa a las haciendas locales.

Por ejemplo, la modificación de la disposición adicional $8 .^{a}$ del TRLHL no se limita al Texto Refundido sino que persigue tres objetivos: por un lado completar las salvedades a la LRBRL; por otro, introducir en el lugar adecuado las excepciones a dos artículos del TRLHL reformados por la LRSAL; y, finalmente, recoger una cláusula general competencial a favor de los Territorios Históricos de colaboración con las entidades locales en materia presupuestaria y financiera.

De estas tres disposiciones únicamente la disposición adicional 1. ${ }^{a}$ de la propia Ley reformadora estaba prevista desde el inicio del procedimiento legislativo. 
Así en el origen de la reforma (anteproyecto y proyecto de ley) sólo se preveía una única disposición adicional $1 .^{a}$ de forma conjunta para Navarra y el País Vasco, en orden a garantizar la salvaguarda de sus competencias históricas sobre régimen local. El proyecto de ley decía así en lo relativo al País Vasco:

«Esta Ley se aplicará a la Comunidad Autónoma del País Vasco en los términos establecidos en el artículo 149.1.14. ${ }^{a}$ y $18 .^{a}$ y disposición adicional primera de la Constitución, sin perjuicio de las particularidades que resultan de la Ley Orgánica 3/1979, de 18 de diciembre, por la que se aprueba el Estatuto de Autonomía para el País Vasco, y de la disposición final tercera de la Ley Orgánica 2/2012, de 27 de abril, de Estabilidad Presupuestaria y Sostenibilidad Financiera» (apartado 2).

Las adiciones efectuadas durante la tramitación legislativa consisten en la adición de la frase "y de las demás normas que actualicen los derechos históricos de los territorios forales» dentro del párrafo 1. ${ }^{\circ}$; en la adición de una segunda parte al párrafo 1. ; y la adición de un nuevo párrafo $2 .^{\circ}$.

Más adelante en su trámite en el Senado, la Ponencia de la Comisión procedió a efectuar una modificación de este párrafo $2{ }^{\circ}$ convirtiéndolo en un apartado 3 independiente con el único objeto de extender su aplicación también a Navarra.

El Pleno del Senado aprobó la división de la disposición adicional en dos diferentes, lo que supuso que el apartado 2 con sus dos párrafos se convirtiera en la nueva disposición adicional $1 .^{a}$ de la LRSAL, pasando el párrafo $1 .^{\circ}$ a ser el apartado 1 y el párrafo $2 .^{\circ}$ el apartado 2.

Por el contrario, la modificación de la disposición adicional 2. ${ }^{a}$ de la $L R B R L$, sobre régimen aplicable a la Comunidad Autónoma del País Vasco, no se encontraba prevista en el proyecto de ley y fue introducida en el trámite de la Comisión con competencia legislativa plena del Congreso de los Diputados. Lo mismo ocurrió con la modificación de la disposición adicional $8 .^{a}$ de la LRBRL. Asimismo se incluyó una disposición adicional $16 .^{a}$ de la LRSAL sobre suministro de información por la Comunidad Foral de Navarra y las Diputaciones Forales del País Vasco, que finalmente no se incorporó a la Ley.

De forma general, cabe destacar que la LRSAL introduce las siguientes novedades:

1. Nuevas salvedades a la aplicación de las nuevas disposiciones de la LRBRL, en especial, en lo que se refiere al sistema competencial impuesto por la LRSAL.

2. Atribución de funciones de tutela financiera a la Comunidad Autónoma del País Vasco, excluyendo o dejando en segundo término la intervención estatal.

3. En algunos casos se establece el órgano interno de la Comunidad Autónoma Vasca competente, en especial, respecto de las Diputaciones Forales y en otros se deja su fijación a la normativa interna de la Comunidad Autónoma Vasca.

Un análisis de las modificaciones introducidas debiera tener en cuenta el conjunto de las tres disposiciones adicionales modificadas o introducidas (D.A. 2. ${ }^{\text {a }}$ LRBRL, D.A. 8. ${ }^{a}$ LHL y D.A. 1. ${ }^{a}$ LRSAL). Por ello, sin perjuicio de que se irá advirtiendo cuál es la disposición referida, el examen de las modificaciones efectuadas en lo que respecta a la Comunidad Autónoma del País Vasco se va a realizar de forma conjunta. 


\section{V.2. Nuevas cláusulas generales de salvedad: la disposición adicional $1 .^{a}$ de la LRSAL y la disposición adicional 8. ${ }^{a}$ delTRLHL}

La LRSAL contiene, en primer lugar, una nueva cláusula de salvaguarda del régimen foral y local vasco, que se adiciona a las ya existentes en el propio texto de la LRBRL y del TRLHL. De esta cláusula cabe destacar su amplitud y generalidad, dado que no se contemplaba en los mismos términos en la LRBRL. Así también hace referencia a la existencia de singularidades que van más allá de las previsiones expresas contenidas en la LRBRL y en el TRLHL, lo que admite una interpretación más amplia. Y, por último, persigue dotar a estas particularidades de permanencia puesto que se contempla su actualización.

En concreto se afirma que "esta Ley se aplicará a la Comunidad Autónoma del País Vasco en los términos establecidos en el artículo 149.1.14. ${ }^{a}$ y $18 .^{a}$ y disposición adicional primera de la Constitución, sin perjuicio de las particularidades que resultan de la Ley Orgánica 3/1979, de 18 de diciembre, por la que se aprueba el Estatuto de Autonomía para el País Vasco, de la disposición final tercera de la Ley Orgánica 2/2012, de 27 de abril, de Estabilidad Presupuestaria y Sostenibilidad Financiera, y de las demás normas que actualicen los derechos históricos de los territorios forales».

La disposición adicional 8. ${ }^{a}$ del TRLHL mantiene la cláusula general de salvaguarda contenida en el anterior texto legal vigente, que aparece copiada en el párrafo $1 .^{\circ}$ de este apartado 1: "Los Territorios Históricos del País Vasco continuarán conservando su régimen especial en materia municipal en lo que afecta al régimen económico-financiero en los términos de la Ley del Concierto Económico, sin que ello pueda significar un nivel de autonomía de las corporaciones locales vascas inferior al que tengan las demás corporaciones locales, sin perjuicio de la aplicación de lo dispuesto en la Ley 7/1985, de 2 de abril, reguladora de las Bases del Régimen Local, y de las competencias que a este respecto puedan corresponder a la Comunidad Autónoma».

La Circular de 11 de marzo de 2014 de la Directora de Relaciones con las Administraciones Locales y Registros Administrativos del Gobierno Vasco afirma, con acierto, que la disposición adicional primera.1 de la LRSAL supone «el reconocimiento de un régimen singular» para su aplicabilidad en la Comunidad Autónoma del País Vasco (p. 6).

\section{V.3. Actualización de denominaciones de los Territorios Históricos}

La disposición adicional 2. ${ }^{a}$ LRBRL actualiza la denominación de los Territorios Históricos a la oficial de Araba/Álava, Gipuzkoa y Bizkaia.

\section{V4. Las competencias de los municipios}

Una de las cuestiones nucleares de la LRSAL es la competencial, dado que incide de forma muy relevante en las competencias de las entidades locales. De forma muy resumida la LRSAL modifica los artículos 7.4, 25, 26, y 27 LRBRL y suprime el conte- 
nido de su artículo 28. La nueva Ley adopta una triple clasificación de las competencias de los municipios: competencias propias, por delegación e impropias (19).

Ello significa que establece un primer nivel de competencias propias de los municipios, más limitado que el contenido en la normativa modificada toda vez que ya no se consagra una cláusula general de competencias (artículo 25.1), sino que se ofrece un listado más reducido del que además se desgajan algunas de ellas a favor de otros niveles superiores (Comunidades Autónomas y Provincias) (artículo 25.2). El artículo 26 reitera el sistema de fijación de servicios mínimos a prestar de forma obligada por los municipios en función de su población, introduciendo una de las novedades más trascendentes cual es que los municipios de menos de 20.000 habitantes pueden ver que la prestación de algunos servicios mínimos sea coordinada por las Diputaciones provinciales, a cuyo efecto éstas elevarán, de conformidad con los municipios afectados, una propuesta de forma de prestación de los servicios coordinados, para su decisión por el Ministerio de Hacienda y Administraciones Públicas. Así pues hay dos tipos de municipios, los municipios «independientes» y los municipios bajo la coordinación de las Diputaciones provinciales. El criterio para fijar esta coordinación se encuentra en el concepto de coste efectivo de los servicios, que se convierte por tanto en el elemento determinante y aparece regulado en el nuevo artículo 116 ter LRBRL.

En segundo lugar, se potencia la posible delegación de competencias por parte de las Comunidades Autónomas o de las provincias en los municipios, asegurándose que la delegación se efectúa con el pago efectivo del coste de dichos servicios.

Finalmente, se limita la intervención de las entidades locales en el ámbito de las denominadas competencias impropias (20). Por un lado, el artículo 7.4 LRBRL indica que las entidades locales "sólo» podrán ejercer estas competencias impropias cuando tengan recursos para ello y además no incurran en duplicidades administrativas, requiriendo para ello de informes favorables de la Administración competente sobre la materia y de la Administración que tenga la tutela financiera. Por otro, desaparecen las "actividades complementarias" del anterior artículo $28 \mathrm{LRBRL}$, ahora suprimido. A ello se unen los límites impuestos por el artículo 86 LRBRL a la iniciativa pública local en materia de actividades económicas.

No procede entrar aquí en el examen de la posible inconstitucionalidad de algunas de las nuevas disposiciones. No obstante, es preciso advertir que el Consejo de Estado ha entendido que los artículos 26.2 y 116 ter pueden ser inconstitucionales por vulnerar el principio de autonomía local (dictamen de 22 de mayo de 2014).

No obstante, la reforma local aprobada queda "desvirtuada» (21) para la Comunidad Autónoma Vasca, como puede verse del juego conjunto de las diversas excepciones introducidas:

(19) Para una visión más general me remito a J. CUESTA REVILLA, "Las competencias municipales. Una aproximación a su nuevo régimen jurídico», en el número monográfico de Cuadernos de Derecho Local sobre "La revisión del Gobierno y la Administración local en la Ley 27/2013», febrero 2014, pp. 98-125.

(20) Ahora incluye también las competencias complementarias del artículo 28 LRBRL suprimido.

(21) Es importante resaltar que de no ser por las disposiciones amparadas en los derechos históricos, la reforma sería plenamente aplicable en la Comunidad Autónoma Vasca. Por tanto no coincido con la afirmación de la Circular de 11 de marzo de 2014 de la Directora de Relaciones con las Administraciones Locales y Registros Administrativos del Gobierno Vasco en la que se afirma que la normativa básica de régimen local no puede desconocer o menoscabar las competencias correspondientes a la Comunidad Autónoma de Euskadi en las materias relacionadas con los diferentes sectores de actuación pública (p. 3). Basta ver, asimismo, las consideraciones al respecto 
1. Competencias propias: La disposición adicional 8. ${ }^{a}$ del TRLHL incluye una salvedad a la aplicación del artículo 25 de la LRBRL. Se excepciona la aplicación del nuevo régimen competencial municipal, puesto que la fijación de las competencias propias queda en manos de las instituciones vascas. Ello significa que los municipios vascos podrán tener un ámbito competencial diferente de los municipios de régimen común. El límite que se impone es el respeto de los criterios establecidos en los apartados 3, 4 y 5 del citado artículo 25 LRBRL.

2. La asignación del ejercicio de competencias a un nivel provincial: la disposición adicional primera de la LRSAL incide en el elemento crucial y determinante de la coordinación de servicios por el nivel superior (de los municipios de menos de 20.000 habitantes a las Diputaciones provinciales) que se fundamenta en la fijación del coste efectivo de los servicios. Si el coste efectivo es mayor, la competencia será objeto de coordinación, y la Diputación repercutirá a los municipios el coste efectivo del servicio en función de su uso (artículo 26.2 LRBRL). Si es menor, la competencia puede quedar en manos del municipio. Y es por ello que el nuevo artículo 116 ter de la LRBRL regula cómo se efectúa el cálculo del coste efectivo de los servicios, para lo que se seguirán los criterios fijados por el Ministerio de Hacienda y Administraciones Públicas. Por un lado, se atribuye a los Territorios Históricos la decisión sobre la forma de prestación de los servicios coordinados, sustrayéndola del Ministerio de Hacienda y Administraciones Públicas (artículo 26.2 LRBRL). Por otro, se advierte que las facultades de coordinación y tutela corresponden (con carácter general) a los Territorios Históricos. Y, en tercer lugar, se recoge la precisión del artículo 26.2 LRBRL de la conformidad de los municipios afectados a la propuesta sobre la forma de prestación de los servicios.

3. El coste efectivo: asimismo se atribuye a la Comunidad Autónoma del País Vasco la aprobación de la metodología de valoración del coste de los servicios transferidos en las materias de las disposiciones adicional 15. ${ }^{a}$ (educación) y transitorias 1. ${ }^{a}$ (salud), 2. ${ }^{a}$ (Servicios sociales) y 3. ${ }^{a}$ (inspección sanitaria). No obstante, se trata de una competencia limitada porque debe atender las directrices y principios que establezca el Ministerio de Hacienda y Administraciones Públicas.

Por otra parte, la nueva redacción de la disposición adicional 2. ${ }^{a}$ da respuesta a diversos elementos relevantes de la LRBRL, como el traspaso de competencias (o la coordinación, que es el término ahora empleado por la $L R B R L$ ) desde los municipios menores a 20.000 habitantes a las Diputaciones provinciales y la minusvaloración de las entidades locales inferiores al municipio. Por un lado, las competencias establecidas en el artículo 26.2 de tutela financiera y control sobre el traspaso competencial referido se atribuyen a las instituciones de la Comunidad Autónoma del País Vasco, dejándose al ámbito interno la determinación de si será competente la Administración autonómica o las Diputaciones forales.

Asimismo la implantación de la coordinación tiene en cuenta como elemento fundamental el coste efectivo de los servicios que se regula en el artículo 116 ter de la

del Dictamen del Consejo de Estado de 22 de mayo de 2014. Es por ello que la disposición adicional octava.1 del TRLHL (en su nueva redacción) no refrenda nada sino que salva lo establecido por la LRSAL. 
LRBRL. Pues bien, también respecto de este precepto, se atribuye a las Diputaciones forales el desarrollo de los criterios de cálculo de dicho coste efectivo, así como la recepción de la comunicación que deben remitir las entidades locales. Debe advertirse que se refiere al desarrollo de los criterios establecidos en el artículo 116 ter, cuando resulta que este precepto indica que la determinación de estos criterios se efectuará por el Ministerio de Hacienda y Administraciones Públicas.

En relación con las transferencias de competencias al nivel autonómico previstas en las disposiciones adicional $15 .^{a}$ y transitorias $1{ }^{a},{ }^{a} .^{a}$ y $3 .^{a}$ de la LRSAL, la disposición adicional 1. ${ }^{a} .2 \mathrm{LRSAL}$ señala que la metodología para valorar su coste corresponde a las instituciones de la Comunidad Autónoma, "atendiendo las directrices y principios que establezca el Ministerio de Hacienda y Administraciones Públicas».

En definitiva, el nuevo panorama legislativo general sobre competencias locales queda exceptuado en la Comunidad Autónoma Vasca, precisando para ello de una nueva regulación autonómica que afiance este "avance» competencial respecto de la situación anterior en relación con la aplicación de la LRBRL y delTRLHL en Euskadi. Si a ello añadimos el principio de continuidad de la disposición transitoria 2. ${ }^{a}$ de la LRBRL, invocado por la Circular de 11 de marzo de 2014 de la Directora de Relaciones con las Administraciones Locales y Registros Administrativos del Gobierno Vasco, las novedades son bien pocas, aunque precisan de inmediato amparo legal a nivel autonómico. Entiendo que el problema principal es salvar el nuevo ámbito competencial del artículo 25.2 de la LRBRL mediante una ley municipal que haga uso de las competencias de salvedad que le reconoce ahora la nueva redacción de la ley estatal.

La Circular distingue entre competencias "antiguas" y "nuevas» a los efectos de lo dispuesto en los artículos 7.4 y 25.2 LRBRL (22). Sin embargo, a mi juicio, lo relevante es la posibilidad que se brinda a la Comunidad Autónoma Vasca de articular un sistema propio y diferente y, además, dotándole de una autonomía interna dado que las decisiones se dejan en el ámbito autonómico vasco (me refiero a los informes del art. 7.4 LRBRL).

\section{V.5. La estructura local: otras entidades locales, regímenes especiales y concejos}

La LRSAL contempla diversas medidas sobre la estructura local, en especial sobre las diversas entidades locales diferentes del municipio. Por un lado, se refiere a las entidades locales menores (artículo 24 bis, supresión del artículo 45, disposición transitoria 4. ${ }^{a}$ todos ellos de la LRBRL), y por otro, a otro tipo de entidades como los consorcios, que reciben una gran atención (disposiciones adicionales $13 .^{a}$ y $14 .^{a}$, transitoria 6. ${ }^{a}$, final 2. ${ }^{a}$ LRSAL), o las mancomunidades (disposición transitoria $11 .{ }^{a}$ LRSAL). También se persigue el redimensionamiento del sector público local (disposición adicional 9. ${ }^{a}$ LRBRL).

(22) La Circular adopta aquí el criterio seguido por gran parte de las normas o circulares autonómicas respecto de la aplicación de la LRSAL. Así por ejemplo pueden verse los artículos 1 y 2 del Decreto-Ley 1/2014, de 20 de mayo, de la Junta de Andalucía, los artículos 3.2 y 6.1 de la Ley 1/2014, de 25 de julio, de la Comunidad de Madrid o la Circular 1/2014, de 28 de abril, de la Dirección General de Administración Local del Gobierno de Aragón. 
Sin embargo, las salvedades introducidas se limitan en la práctica solamente a las entidades locales inferiores al municipio. Así, aunque se configure como una medida de tutela financiera, es obligado incluir aquí la referencia a las entidades locales inferiores al municipio o concejos. Se trata de una figura denostada por la LRSAL, pero que tiene una gran relevancia en el País Vasco, sobre todo, en el Territorio Histórico de Álava (23).

La LRSAL persigue la reconversión de las entidades locales menores en órganos desconcentrados (artículo 24 bis LRBRL y supresión del artículo 45 LRBRL), aunque se ve obligada a mantener la personalidad jurídica de aquellas ya existentes (disposición transitoria 4. ${ }^{a}$ LRBRL).

Desde el ámbito de la Comunidad Autónoma Vasca, se pretende excluir esta posición y sobre todo, que su posible aplicación se mantenga en la órbita foral. Por ello se introduce una salvedad competencial a favor de las Instituciones Forales:

"Igualmente, de acuerdo con lo previsto en la disposición transitoria cuarta de la Ley $27 / 2013$ de racionalización y sostenibilidad de la Administración Local, las entidades de ámbito territorial inferior al municipio comunicarán a las Instituciones Forales sus cuentas y serán estas Instituciones Forales quienes acuerden su disolución si así procede en aplicación de la mencionada disposición» (D. A. 2. ${ }^{a}$.6 LRBRL).

Por consiguiente, la supresión de concejos, por esta vía de disolución-sanción por la no presentación de las cuentas en plazo, queda en manos de la Diputación Foral. Pero, incluso, la competencia general a favor de la Comunidad Autónoma Vasca permite concluir que la estructura de los Concejos se mantiene dentro de la órbita competencial de los Territorios Históricos, fuera de la aplicación de las normas generales modificadas, suprimidas o adicionadas por la LRSAL respecto de las entidades locales inferiores al municipio.

Por otra parte, el apartado 1 de la disposición adicional 2. ${ }^{a}$ LRBRL mantiene el texto original en cuanto a la singularidad de los Territorios Históricos que se encuentra en íntima relación con lo dispuesto en su artículo 39 (no modificado). Ahora en este apartado 1 se incluye una frase "amparando y garantizando, asimismo, las peculiaridades históricas de las Entidades Locales de sus territorios", que hace referencia a un mandato a los Territorios Históricos que deberán respetar dichas peculiaridades de los entes locales.

\section{V.6. Tutela financiera}

Ya se ha advertido más arriba de la inescindible unidad entre régimen local y haciendas locales y, en especial, la vinculación de la LRSAL con el artículo 135 CE y con la LOEPSF. Se persigue la reducción del gasto público, no sólo el equilibrio presupuestario que exige la CE. Por eso la LRSAL está plagada de medidas tendentes a reducir el gasto público local, desde la perspectiva de una gran desconfianza en la actuación local. Ello provoca que se prevean nuevas medidas de tutela financiera, en ratificación y adición de las ya establecidas por la LOEPSF.

(23) El Territorio Histórico de Álava aprobó la Norma Foral 11/1995, de 20 de marzo, de Concejos. Véase la obra colectiva El Concejo alavés ante el siglo XXI, ed. Juntas Generales de Álava, Vitoria-Gasteiz 2003. 
Y evidentemente al hablarse de medidas financieras, aparece aquí la posición singular de la Comunidad Autónoma Vasca en relación con el Estado que se asienta en el Concierto Económico. Ya así lo preveía con anterioridad la LOEPSF, tal como se ha indicado más arriba.

Por eso cabe dudar, como hace la Circular de 11 de marzo de 2014 de la Directora de Relaciones con las Administraciones Locales y Registros Administrativos del Gobierno Vasco (24), de su aplicación en la Comunidad Autónoma Vasca.

El apartado 5 de la disposición adicional 2. ${ }^{a}$ LRBRL recoge la cita expresa de la Ley del Concierto Económico (Ley 12/2002, de 23 de mayo) (25), que se convierte en el paraguas protector frente a la entrada de la Administración estatal en las entidades locales vascas. Este nuevo precepto se refiere a las competencias en materia de tutela financiera establecidas en los artículos 7.4 y 26.2 de la LRBRL que se atribuyen a la Comunidad Autónoma Vasca, dejando la fijación del órgano competente para ello a la regulación interna (26). El criterio determinante para establecer la Administración competente en el ámbito interno es el artículo 48.5 de la Ley del Concierto Económico citado expresamente, que dice así: "Las facultades de tutela financiera que, en cada momento, desempeñe el Estado en materia de Entidades locales, corresponderán a las instituciones competentes del País Vasco, sin que ello pueda significar, en modo alguno, un nivel de autonomía de las Entidades locales vascas inferior al que tengan las de régimen común».

Se trata, nuevamente, de una combinación entre competencia foral y respeto de la autonomía local.

Además se introducen cuatro salvedades concretas:

1. Control del plan económico y financiero. El artículo 116 bis de la LRBRL introduce unas reglas específicas respecto del plan económico y financiero que deben aprobar y seguir las entidades locales que incumplan el objetivo de estabilidad presupuestaria, conforme establece la LOEPSF, concediendo a las diputaciones provinciales un papel colaborador en las decisiones que corres-

(24) La circular afirma lo siguiente: "Las peculiaridades del sistema de financiación propio del Concierto Económico, unido a las previsiones contenidas en la disposición adicional octava del TRLHL, así como las singularidades competenciales e institucionales existentes en la Comunidad Autónoma de Euskadi, ponen en duda la aplicabilidad de dichos mandatos en el sistema vasco. Aun así, y en aras de dotar de seguridad a nuestro marco jurídico, se considera pertinente un expreso pronunciamiento normativo que detalle la distribución competencial en dichas materias, entre los distintos niveles de Administración de la Comunidad Autónoma Vasca, pronunciamiento que deberá ser contenido en la Ley Municipal de Euskadi» (p. 6).

(25) Véase F. DE LA HUCHA CELADOR, El régimen jurídico del Concierto Económico, Ed. Ad Concordiam, Bilbao, 2006, en especial, pp. 95-101, en las que explica las potestades armonizadoras del País Vasco en relación con las Haciendas locales.

(26) Téngase en cuenta la posición diversa que ha mantenido la Comisión Arbitral respecto de la asignación de las competencias sobre tutela financiera en el nivel interno, es decir, sobre si éstas corresponden a las Instituciones Comunes o a los Territorios Históricos.

En un primer momento se pronunció a favor de la competencia de los Territorios Históricos para la regulación del régimen presupuestario y de contabilidad de las entidades locales (Decisión 2/2003, de 17 de marzo, sobre el Proyecto de Norma Foral Presupuestaria de las Entidades Locales del Territorio Histórico de Bizkaia, publicada en el BOPV de 20 de octubre de 2003). Más adelante, por el contrario, reconoció la competencia de las Instituciones Comunes sobre el régimen financiero de las entidades locales, limitando el ámbito de intervención de los Territorios Históricos de forma muy significativa (Decisión 3/2012, de 19 de abril, sobre el Proyecto de Ley Municipal de Euskadi, publicada en el BOPV de 13 de junio de 2012). Debe advertirse que ambas resoluciones contaron con varios votos particulares en su contra. 
ponde adoptar a la Administración que ejerza la tutela financiera. Pues bien, lo que se trata ahora es de reconocer a las Diputaciones Forales el papel de Administración decisoria atribuyéndoles la tutela financiera. Es por ello que las Diputaciones Forales aprobarán los planes económico-financieros y supervisarán su cumplimiento. Únicamente se les impone que esta aprobación, que les permite concretar las reglas necesarias para la formulación de estos planes, se efectúe "de conformidad con la normativa dictada al efecto por el Estado» (disposición adicional 2. ${ }^{a} .6$, párrafo $3 .^{\circ}$ LRBRL).

2. Información sobre derechos de difícil o imposible recaudación. La disposición adicional 8. ${ }^{a}$ del TRLHL en su apartado 2 pretende obviar la competencia del Ministerio de Hacienda y Administraciones Públicas recogida en el nuevo artículo 193 bis TRLHL. Así pues las entidades locales vascas deberán informar a los Territorios Históricos del resultado de aplicación de los criterios determinantes de los derechos de difícil o imposible recaudación (disposición adicional 8. a .2 TRLHL).

3. Los controles de intervención: se remitirán a las Diputaciones Forales las discrepancias entre los órganos municipales y el interventor municipal. Los municipios vascos deberán enviar a las Diputaciones forales tanto los informes de los órganos interventores como los informes justificativos del Alcalde sobre su actuación. Seguidamente se fija al Tribunal Vasco de Cuentas Públicas como primer estadio de control de la actuación en el caso de resoluciones y acuerdos locales discrepantes sobre los informes de intervención, al que deben remitirse los informes del artículo 218 TRLHL, aunque se advierta que lo será sin perjuicio de las competencias del Tribunal de Cuentas (disposición adicional 8. ${ }^{a} .2$ TRLHL).

4. Convenios de colaboración: el nuevo apartado 3 de la disposición adicional 8. ${ }^{\text {a }}$ del TRLHL atribuye a las Diputaciones Forales la competencia para «formalizar convenios con las Entidades Locales para reforzar la autonomía y eficacia de los órganos responsables del control y fiscalización interna de la gestión económico-financiera, contable y presupuestaria de las citadas Entidades Locales». En definitiva, se recupera la competencia prevista en la disposición adicional $7 .^{a}$ LRSAL.

Habida cuenta de estas salvedades, parece correcto concluir que la información en materia de tutela financiera (disposición adicional 12. ${ }^{a}$ LRSAL) habrá de ser ofrecida por las Diputaciones Forales.

Por el contrario, no hay previsiones sobre una posible aplicación específica de la modificación introducida en el artículo 36.1 de la Ley 2/2011, de 4 de marzo, de Economía Sostenible (disposición final 3. ${ }^{a}$ LRSAL) en caso de incumplimiento de remisión de información al Ministerio de Hacienda y Administraciones Públicas y al Tribunal de Cuentas.

\section{V.7. Funcionarios con habilitación de carácter nacional}

Desde su origen, la LRBRL recogía las competencias de la Comunidad Autónoma Vasca en materia de funcionarios con habilitación de carácter nacional que posterior- 
mente se había introducido en el EBEP, al derogar éste los preceptos de la LRBRL. Ahora con motivo de la vuelta desde el EBEP a la LRBRL en su nuevo artículo 92 bis, se actualiza la disposición adicional 2. ${ }^{a}$ LRBRL con la cita del nuevo precepto ahora vigente. La regulación había salvaguardado siempre la competencia sobre convocatoria de concursos, nombramientos, formación y baremo de los concursos de estos funcionarios.

Las competencias para la fijación del baremo aparecen distribuidas entre el Estado (80\%), la Comunidad Autónoma (15\%) y la entidad local (5\%). La modificación consiste en que el porcentaje atribuido a las instituciones competentes de la Comunidad Autónoma Vasca es ahora del 15\% (antes el 10), y el de la corporación local interesada el $5 \%$ (igual que el resto de las entidades locales, aunque antes era el $25 \%)$.

No obstante, el nuevo apartado 7 parece contener un alcance mayor que el del texto anterior. La nueva redacción de este apartado ya no se limita a los concursos y nombramientos, que pasan a ser una competencia mínima que siempre les ha correspondido, sino que contiene una referencia más general a "todas las facultades previstas» en "la normativa reguladora» de los funcionarios con habilitación de carácter nacional.

Así pues, todas estas facultades se conceden a las instituciones de la Comunidad Autónoma Vasca, que luego deslindará su atribución en el ámbito interno (así se dice, «en los términos que establezca la legislación autonómica»).

\section{V.8. Retribuciones de cargos, personal eventual y funcionarios}

El objetivo de reducción del gasto público que imbuye a la LRSAL se presenta asimismo en relación con los gastos de los cargos públicos locales y el personal al servicio de las entidades locales. Se parte de que las entidades locales constituyen una organización "sobredimensionada" y que por tanto es preciso recortar.

La LRSAL modifica diversos preceptos de la LRBRL para lograr dicho objetivo. Por un lado, se fijan límites retributivos para los miembros de las entidades locales en el nuevo artículo 75 bis. Por otro, se introducen límites al número de miembros con dedicación exclusiva (nuevo artículo 75 ter). En tercer lugar, se retocan asimismo las normas sobre el personal eventual y directivo de las entidades locales (artículo 104 bis).

Se deja en manos de los Territorios Históricos la fijación de los "límites máximos totales del conjunto de las retribuciones y asistencias de los miembros de las Corporaciones Locales, del personal eventual y del resto de personal al servicio de las Corporaciones Locales y su sector público y de los funcionarios con habilitación de carácter nacional» (apartado 11 de la disposición adicional 2. ${ }^{a} \mathrm{LRBRL}$ ).

El límite para esta competencia se encuentra en que "la determinación de tales retribuciones atenderá a los principios y estructura establecidos, en su caso, por la legislación estatal».

Téngase en cuenta la aprobación del Real Decreto Ley 1/2014, de 24 de enero, que establece el régimen retributivo de los miembros de las Corporaciones Locales (artículo 11.3 del Real Decreto Ley). 


\section{Papel de las instituciones comunes y de los territorios históricos en el desarrollo de la reforma local}

Como se ha resaltado más arriba, el marco general de distribución competencial en el nivel interno de la Comunidad Autónoma Vasca se encuentra en el EAPV, en la LTH y en la Ley del Concierto Económico (27).

Conviene ahora detenerse en las disposiciones de la LRSAL que definen las competencias del nivel interno de la Comunidad Autónoma Vasca o bien dejan para su posterior distribución por la normativa propia de la Comunidad Autónoma. La reforma local de 2013 deja pendientes algunas cuestiones de orden interno, aunque asimismo resuelve otras generalmente a favor de los Territorios Históricos. Así lo destaca la Circular de 11 de marzo de 2014 de la Directora de Relaciones con las Administraciones Locales y Registros Administrativos del Gobierno Vasco al indicar que las nuevas disposiciones derivadas de la LRSAL "establecen un complejo sistema de atribuciones puntuales de determinadas facultades o competencias sobre ámbitos materiales o espacios de intervención específicos a diferentes instituciones vascas» (p. 7).

Así en primer término deja en poder de los Territorios Históricos todo lo relativo a su autoorganización y funcionamiento, así como lo relativo a su régimen económico financiero. Cabe referirse a los siguientes supuestos previstos expresamente en la LRSAL:

- Funcionamiento y ejercicio de las competencias por el Territorio Histórico (disposición adicional 2. ${ }^{a} \mathrm{LRBRL}$, apartados $1,2,3,4$ y 5, primer párrafo).

- Gestión económico-financiera y presupuestaria del propio Territorio Histórico (disposición adicional 2. ${ }^{a}$ LRBRL, apartado 10).

Asimismo se atribuyen de forma expresa a los Territorios Históricos (o a las Diputaciones Forales) las siguientes competencias:

- Determinación de la forma de prestación de los servicios del artículo 26. 2 LRBRL (disposición adicional 1. '.1 LRSAL).

- Régimen económico-financiero de las entidades locales, que incluye el contenido del artículo 116 ter (coste efectivo), del artículo 116 bis (tutela financiera) y de la disposición transitoria 4. ${ }^{a}$ de la LRBRL (disolución de los concejos), previsiones realizadas por la Disposición adicional 2. ${ }^{a}$.2 LRBRL.

- Funciones de tutela financiera y recepción de informes de los artículos 193 bis y 218 TRLHL (disposición adicional 2. a 11 LRBRL).

- Fijación de límites máximos de retribuciones y asistencias (disposición adicional 8. . $3 \mathrm{TRLHL}$ ).

- Celebración de convenios con municipios (disposición adicional 8. ${ }^{a}$.3 TRLHL).

Por otra parte, la atribución de competencias propias a los municipios (art. 25 LRBRL) se deja en manos bien de la CAPV o de los TTHH en función de sus respectivas competencias (disposición adicional 8. ${ }^{a} .1$ párrafo 2. ${ }^{\circ} \mathrm{TRLHL}$ ).

(27) Sobre las competencias de los Territorios Históricos y su regulación en la LTH véase S. LARRAZABAL BASAÑEZ, "¿26 años no es nada...? Luces y sombras en la distribución de competencias entre las instituciones comunes de la Comunidad Autónoma del País Vasco y las de sus Territorios Históricos», en Revista Vasca de Administración Pública núm. 87-88/2010, pp. 707-742. 
Por el contrario quedan indeterminadas («las instituciones competentes»), dejándose su atribución al ordenamiento interno del País Vasco, las siguientes competencias:

— Fijación del coste de los servicios transferidos (disposición adicional 1. ${ }^{a} .2$ LRSAL).

— Las funciones de los artículos 7.4 y 26.2 atribuidas a la Administración (disposición adicional $\left.2 .^{\text {a }} .5 \mathrm{LRBRL}\right)$.

- Las competencias sobre funcionarios con habilitación de carácter nacional (disposición adicional 2. ${ }^{a} .7$ y 8 LRBRL).

Finalmente, a favor de las Instituciones Comunes solamente se deja la realización del Convenio del IVAP (disposición adicional 2. ${ }^{a}$.9 LRBRL).

\section{VII. ¿Por fin, una Ley municipal de Euskadi?}

Es evidente que la reforma introducida por la LRSAL para nada afecta a los Territorios Históricos, en cuanto instituciones propias de la Comunidad Autónoma del País Vasco y diferentes de las provincias. Y ello, a pesar de que les correspondan por defecto las competencias propias de las Diputaciones provinciales. Se trata de una cuestión hoy manifiestamente clara y que no tiene discusión, y que ha recibido confirmaciones rotundas tanto a nivel legislativo como a nivel jurisprudencial, tal como se ha expuesto más arriba. Se trata de un camino que ya ha culminado y que no tiene vuelta atrás: las Diputaciones Forales (y mucho menos las Juntas Generales) no son entes locales. Así pues, lisa y llanamente, cabe concluir que se encuentran fuera del ámbito de la reforma local efectuada por la LRSAL.

Simplemente puede decirse que los Territorios Históricos han visto ampliadas sus competencias de una forma indirecta, por la ampliación competencial efectuada a favor de las diputaciones provinciales. Además, aumentarán sus competencias por la vía del control sobre los municipios. Ya se ha resaltado la gran desconfianza que la LRSAL muestra respecto de los municipios, lo que le hace diseñar nuevos instrumentos de control, sobre todo de tutela financiera, o cuando menos profundizar en los ya existentes. Por esta vía se produce una ampliación competencial de los Territorios Históricos que reciben un nuevo conjunto de funciones bien de colaboración (por ejemplo en los amplios términos el apartado 3 de la disposición adicional $\left.8{ }^{a}{ }^{\mathrm{T}} \mathrm{TRLHL}\right)$ como de control (recepción de informes, informes preceptivos e incluso vinculantes a emitir, etc.).

Fuera de ello, la reforma local de 2013 se centra en el nivel estrictamente municipal, así como en el inframunicipal (concejos) o supramunicipal (mancomunidades, agrupaciones y consorcios). Y, como he referido más arriba introduce nuevas salvedades a favor de la Comunidad Autónoma Vasca que es preciso consolidar cuanto antes, dado que ha quedado perfilado, a partir de 2013, un régimen diferencial más acusado.

Los Territorios Históricos han venido aprobando diversas Normas Forales y reglamentos en materia local. Por ejemplo, cabe resaltar las Normas Forales del Territorio Histórico de Álava que afectan a su régimen local: Concejos, Elección de Regidores y Vocales de los Concejos, Hermandades, Cuadrillas, Haciendas locales, Fondo Foral de Financiación de las Entidades locales, presupuestaria de las entidades locales y de- 
marcaciones territoriales. El de Bizkaia ha aprobado Normas forales sobre demarcaciones territoriales, haciendas locales, entidades de ámbito supramunicipal y presupuestaria. Y el de Gipuzkoa cuenta asimismo con Normas forales sobre demarcaciones territoriales, haciendas locales, Fondo foral de financiación municipal, entidades de ámbito supramunicipal, presupuestaria e, incluso, la ya citada Norma Foral 1/2013, de 8 de febrero, sobre tutela financiera de los municipios.

Sin embargo hasta el momento no se ha aprobado la Ley municipal de Euskadi, a pesar de que han existido varios intentos que no han tenido éxito (28) y de que se considera una ley imprescindible(29). El carácter necesario de esta Ley municipal viene ahora resaltado por la Circular de 11 de marzo de 2014 de la Directora de Relaciones con las Administraciones Locales y Registros Administrativos del Gobierno Vasco, cuya conclusión 7. a afirma que "el elemento de cierre de este modelo será la futura Ley Municipal de Euskadi», resaltando la provisionalidad de las medidas que se adopten hasta su aprobación.

Acaba de aparecer publicado un Anteproyecto de Ley Municipal de Euskadi, de fecha 1 de julio de 2014, que se somete a información pública. Y en el mismo se califica su aprobación como inaplazable, tanto para desterrar la precariedad del marco normativo institucional como para desarrollar el «notable catálogo de especialidades para el caso vasco» derivadas de la reforma de 2013.

Sólo una regulación legal permite adoptar un modelo local vasco propio y diferenciado del común. Más aún, cabe decir si se quiere redireccionar el timón para que los municipios vascos puedan salir de la atonía y limitaciones en que la reforma de 2013 ha sumido a los municipios españoles.

Y, entiendo, que éste es el espíritu del Anteproyecto de Ley Municipal de Euskadi, iniciar una vía nueva caracterizada por tres líneas maestras:

1. Integrar al municipio dentro del entramado institucional autonómico como una parte más, en iguales condiciones, que las Instituciones comunes y los Territorios Históricos. Es, por ello, que se afirme que esta Ley municipal supone el cierre del proceso de institucionalización interna de Euskadi.

2. Reforzamiento del municipio, afirmando su posición central, dotándole de poderes y competencias, articulando canales de participación, y dotándole de visibilidad institucional.

3. Para la efectividad de la autonomía local, se garantiza la sostenibilidad financiera, a fin de que el municipio pueda actuar autónomamente y además prestar servicios de calidad a los ciudadanos.

El Anteproyecto hace "uso" de las salvedades de la aplicabilidad de la LRBRL, del TRLHL y de la LRSAL a la Comunidad Autónoma del País Vasco. Basta para ello observar algunos aspectos destacados del Anteproyecto, más allá de los objetivos y principios tan ambiciosos que enuncia. Son los siguientes:

(28) Para una visión más amplia, me remito a J. M. ENDEMAÑO ARÓSTEGUI, «La regulación del régimen local en la Comunidad Autónoma del País Vasco. Una cuestión pendiente», en Revista Vasca de Administración Pública, núm. 96/2013, pp. 111-152.

(29) Así la califica CASTELLS ARTECHE, ofreciendo algunas ideas al respecto ( LLos regímenes especiales de la Comunidad Autónoma del País Vasco", cit., pp. 4417-4423). 
a) El sistema de competencias se separa totalmente de lo dispuesto en la LRBRL. Por un lado se posiciona a favor de un amplio número de competencias propias, considerando las competencias transferidas o delegadas como una vía excepcional. Así, por un lado, su artículo 16 consagra la cláusula universal de competencias municipales; por otro, su artículo 17 recoge nada menos que 37 ámbitos materiales para el establecimiento de competencias municipales; y admite las competencias impropias con total amplitud en su artículo 14.4 y también la iniciativa local en el ejercicio de actividades económicas en su artículo 87.

b) La estructura local se sostiene sobre el municipio (artículo 8). Pero también se reconocen como entidades locales a los Concejos, Hermandades y Cuadrillas de Álava e, incluso, se prevé la existencia de entidades locales menores, con atribución del control a los Territorios Históricos (artículos 12 y 13). Por otra parte, se produce un renacimiento del principio de autoorganización municipal, que se plasma tanto en el modo de gestión de los servicios públicos locales como en el sistema de entidades locales intermedias.

c) No se dejan de lado dos principios básicos: la racionalidad administrativa y la sostenibilidad financiera. El primero se concreta en diversas disposiciones que tratan de evitar la duplicidad (incluso se hace referencia a la triplicidad) de estructuras administrativas, así como la relación directa con los ciudadanos por el ámbito más cercano a ellos o en el que el servicio se preste de forma más eficiente. Y la sostenibilidad financiera aparece como agua subterránea que brota en los diversos títulos del Anteproyecto para culminar con su último Título, el IX, sobre financiación municipal.

Además, el Anteproyecto no olvida los elementos de detalle que aparecen en las salvedades arriba enunciadas. Simplemente ahora estos elementos de detalle, sobre todo de intervención de las Instituciones Comunes o de los Territorios Históricos, cobran un nuevo sentido diluidos en una música bien diferente.

Finalmente, debo advertir que me he limitado a exponer los aspectos del Anteproyecto de Ley Municipal de Euskadi más relacionados con la reforma local general de 2013. El Anteproyecto es más ambicioso y aborda muchas otras cuestiones de gran relevancia e interés, pero no es éste el lugar de su examen. Y asimismo el Anteproyecto da por hecho que sólo debe limitarse a las competencias autonómicas sobre régimen local, que son completadas por las que corresponden a los Territorios Históricos.

Trabajo recibido el 8 de septiembre de 2014.

Aceptado por el Consejo de Redacción el 10 de octubre de 2014. 
LABURPENA: Lan honetan, 2013ko abenduan Espainian egindako tokiko erreforma azaltzen da. Erreforma hori sakona izan da, eta toki-araubideko funtsezko alderdietan eragin du; esaterako, eskumenetan eta toki-jardueren kontrolean. Azterketa toki-erregimenarekin lotutako eskubide historikoen ondoriozko salbuespenen azterketa du ardatz. Kontzertu ekonomikoa da salbuespen horien oinarria; hori dela-eta, are bereziagoak dira eta Euskal Autonomia Erkidegoak aukera du toki-araubideko eredu propioa sortzeko. Ondorio gisa, adierazten da Euskadiko udal-legea berehala onartu behar dela eredu hori lortzeko.

HITZ GAKOAK: Tokiko erreforma. Udal-autonomia Eskubide historikoak. Udal-legea. Udal-eskumenak.

RESUMEN: El estudio expone la reforma local efectuada en España en diciembre de 2013, que ha sido de gran calado, afectando a aspectos nucleares del régimen local como son las competencias y el control de las actuaciones locales. Y se centra en el análisis de las excepciones derivadas de los derechos históricos relativos al régimen local, articuladas también en torno al Concierto Económico, lo que acrecienta su singularidad y permite a la Comunidad Autónoma Vasca diseñar un modelo local propio. Se concluye que para ello es necesario que se proceda a la inmediata aprobación de la Ley municipal de Euskadi.

PALABRAS CLAVE: Reforma local. Autonomía municipal. Derechos históricos. Ley municipal. Competencias municipales.

ABSTRACT: The study sets forth the local reform carried out in Spain in December 2012, which was a major one, affecting some core aspects of the regime of local entities as competences and the monitoring of local actions. And it focuses on the analysis of the exceptions that derive from the historical laws regarding the local regime, articulated also around the Economic Concert, which makes its singularity bigger and it allows the Basque Autonomous Community to design its own model of local entities. It concludes that in order to do so it is necessary to inmediately pass an Act on municipalities for Euskadi.

KEYWORDS: Local reform. Municipal autonomy. Historical Laws. Municipal Act. Municipal competences. 